\title{
Prevalence, Semiology, and Risk Factors of Epilepsy in Alzheimer's Disease: An Ambulatory EEG Study
}

\author{
András Horváth ${ }^{\mathrm{a}, \mathrm{b}, *}$, Anna Szúcs ${ }^{\mathrm{b}}$, Zoltán Hidasic ${ }^{\mathrm{c}}$ Gábor Csukly ${ }^{\mathrm{c}}$, Gábor Barcs ${ }^{\mathrm{b}}$ \\ and Anita Kamondi, ${ }^{\text {b }}$ \\ ${ }^{a}$ Department of Anatomy, Histology and Embryology, Semmelweis University, Budapest, Hungary \\ ${ }^{\mathrm{b}}$ National Institute of Clinical Neurosciences, Hungary \\ ${ }^{\mathrm{c}}$ Department of Psychiatry and Psychotherapy, Semmelweis University, Budapest, Hungary \\ ${ }^{\mathrm{d}}$ Department of Neurology, Semmelweis University, Budapest, Hungary
}

Accepted 13 March 2018

\begin{abstract}
.
Background: Alzheimer's disease (AD) is the primary cause of cognitive decline. A growing body of evidence suggests that AD patients have a higher risk to develop epileptic seizures; however, results are contradictory due to different methodological approaches of previous studies.

Objective: We aimed to identify the prevalence, semiology, and risk factors of epilepsy in AD using long-term EEG.

Methods: We selected forty-two AD patients and examined them using 24-hour ambulatory EEG. Neurological and epileptological data were collected with retro- and prospective methods. We analyzed the semiology of the identified seizures and the possible risk factors using logistic regression analysis.

Results: We identified seizures confirmed by EEG in $24 \%$. The majority of the seizures were aware focal (72\%) without any motor activity (55\%). We found epileptiform discharges without seizures in $28 \%$. Patients with seizures and only with epileptic EEG activity showed similar clinical and demographical features. Higher education (OR:1.8) and lower Addenbrooke Examination Score (OR: 0.9) were identified as risk factors of epilepsy. Increase of 0.1 point in the VerbalLanguage/Orientation-Memory ratio (VLOM) was associated with higher epilepsy risk as well (OR:2.9).

Conclusion: Epilepsy is a frequent comorbidity of AD. Since most of the seizures are aware non-motor focal seizures, sensitive EEG techniques are required for precise diagnosis of epilepsy. Long-term ambulatory EEG is a safe and welltolerated option. Epileptiform EEG in AD signals the presence of concomitant epilepsy. Clinicians have to pay attention to comorbid epilepsy in dementia patients with high education, with high VLOM ratio and severe stage.
\end{abstract}

Keywords: Alzheimer's disease, epilepsy, long-term EEG, seizures, semiology, risk factors

\section{INTRODUCTION}

Major neurocognitive disorders are devastating conditions leading to progressive cognitive deterioration and affecting approximately 47 million people worldwide [1]. Expectedly, this number will triple

\footnotetext{
*Correspondence to: András Horváth, MD, National Institute of Clinical Neurosciences, 57 Amerikai út, 1145-Budapest, Hungary. Tel.: +36305421019; E-mail: andras.horvath.semmel weis@gmail.com.
}

by 2050 [2]. The most common cause of cognitive decline is Alzheimer's disease (AD) which is an important mortality factor severely compromising the quality of life of the elderly [3]. While numerous studies identified important contributing genes, molecules and factors in the development of $\mathrm{AD}$, we are still not able to treat or significantly slow down the progression of cognitive decline. This is why studies focusing on the possibly modifiable risk factors are gaining more attention [4]. While the concomitant 
cardiovascular disorders and metabolic conditions are frequently analyzed in $\mathrm{AD}$, the real prevalence and impact of epilepsy is still unclear [5].

Epilepsy and $\mathrm{AD}$ share many similar patterns including neuropathological hallmarks, neuroimaging findings and molecular changes. The most frequently known protein aggregations of $\mathrm{AD}$ such as the amyloid plaques and tau neurofibrills have been demonstrated in the samples of mesio-temporal lobe epilepsy patients (MTLE) as well [6, 7]. Temporal hypometabolism shown by PET imaging and hippocampal sclerosis revealed by MRI scans are shared features $[8,9]$. The decrease in calbindin-D28 expression in the dentate gyrus indicating pathological hyperactivity is a similar finding [10]. Genetically modified animal models of AD frequently have epileptic seizures even in their early phase of life $[11,12]$.

Human prevalence studies on AD-related epileptic seizures demonstrated highly variable results ranging between $0.5-64 \%$ [5]. However, we cannot convincingly compare the reported prevalence values due to the different methodological approaches used in various studies. Prospective studies and postmortem histological confirmation of the diagnosis were rare among investigations reporting about epilepsy prevalence in AD [13-16]. Another important obstacle is that the diagnostic criteria of $\mathrm{AD}$ have significantly changed over the last decades [17]; hampering conclusions from the early studies. Additionally, studies examined AD patients with different severity of cognitive impairment making problematic the comparisons of incidence rates. The lack of EEG recordings in most studies is an additional important limiting factor. Based on the current International League Against Epilepsy (ILAE) guidelines, epilepsy is diagnosed if the patient suffered two independent epileptic seizures or only one but the EEG shows interictal epileptiform discharges (IEDs) [18]. Therefore, the diagnostic accuracy of any study without adequate EEG recordings is certainly reduced. While numerous studies $[19,20]$ used different EEG techniques and analysis (e.g., directionality of synchronization, resting state source analysis, spectral and power analysis, spatial coupling, functional connectivity measurements) to identify the biomarkers of $\mathrm{AD}$ (for a thorough review, see [21]), unfortunately, EEG is barely applied in AD studies focusing on comorbid epilepsy [5]. Furthermore, analysis of the semiological features and risk factors of seizures was conducted only in 3 studies [22-24]. Based on these results, AD-associated epileptic seizures are nonmotor aware focal seizures occurring predominantly in advanced disease stages. Because epileptic activity might have an important role in the pathophysiology of $\mathrm{AD}[25,26]$, there is a clear need for further and more detailed studies on the prevalence of epilepsy in $\mathrm{AD}$ [27].

The objective of this study was to examine rigorously selected AD patients by a sensitive EEG method to analyze the prevalence of epilepsy, the semiology of epileptic seizures and identify their possible risk factors.

\section{METHODS}

\section{Patient selection}

In the framework of the Hungarian National Brain Research Program, we recruited 42 AD patients (25 women) treated in the Department of Neurology of the National Institute of Clinical Neurosciences in Budapest, Hungary. The patients met the revised diagnostic criteria of the National Institute of Neurological and Communicative Disorders and Stroke and Alzheimer's disease and Related Disorders Association (NINCDS-ADRDA) for probable AD [17]. Since we have no access to PET biomarkers and limited access to CSF biomarker analysis in our country, we selected the bitemporal, bifrontal, and hippocampal atrophy on the structural MRI as supporting biomarkers, as they are equally accepted biomarkers of $\mathrm{AD}$ according to the current guidelines. MRI acquisition was obtained in every case and images were analyzed visually by experienced radiologists and trained physicians independently. Medial temporal lobe atrophy (MTA) score was applied to support the diagnosis of AD [28]. MTA analyzes T1 weighted MRI images through the hippocampus at the level of the anterior pons and examines three features: width of the choroid fissure, width of the temporal horn of the lateral ventricle and the height of the hippocampus [28]. The score ranges between 1 and 4; atrophy is significant and the presence of $\mathrm{AD}$ is likely if the score $\geq 2$. All known seizure provoking factors were used as exclusion criteria: antipsychotic, benzodiazepine, antihistamine or antidepressant therapy in the last month; MRI lesions other than the typical features of $\mathrm{AD}$ including vascular lesions [29]; alcohol or drug dependency, CNS infection, head trauma with loss of consciousness, stroke, major depression, schizophrenia, electroconvulsive therapy, untreated underactive or overactive thyroid, liver or kidney failure in the medical history. Because it is known that epileptic seizures may precede the onset 
of cognitive decline by a few years [30], we excluded patients with a seizure-history longer than 10 years prior to the onset of cognitive decline. The patients underwent proper physical and epilepsy evaluation including medical, neurological status, epilepsy history and seizure description, provoking factors, etc.; routine blood checks including thyroid functions. Besides demographic markers we recorded the onset of cognitive decline, the duration of dementia, the first appearance of seizure-like symptoms, the handedness and education-level of the patients as well as the use of anti-dementia medication. We obtained informed consent from each patient. Our research was authorized by the National Ethic Committee (024505/2015/OTIG).

\section{Neuropsychology}

A neuropsychological testing was performed in each case by a trained clinician or qualified neuropsychologist. We selected the Addenbrooke Cognitive Examination (ACE) for measuring cognitive functions. ACE is a frequently applied bedside neuropsychological test battery for the diagnostic evaluation of dementia and mild cognitive impairment. It is highly sensitive (94\%) and specific (89\%) for $\mathrm{AD}$ [31]. It measures five cognitive domains: verbal fluency, language, memory, visuo-spatial and executive-orientation skills. The maximum score is 100; a score less than 82 indicates dementia with $84 \%$ sensitivity and $100 \%$ specificity without age and education dependency. An important advantage of ACE is that it contains the Mini-Mental State Examination (MMSE) score as well. With MMSE, clinicians can assess the severity of dementia and compare the results with other studies because this is the most frequently applied diagnostic test in the evaluation of cognitive decline [32]. Furthermore, the ACE VLOM ratio ((Verbal fluency+ Language scores)/ (Orientation and Memory scores)) can differentiate AD and frontotemporal dementia [33]; a VLOM ratio $>3.2$ indicates $\mathrm{AD}$ type dementia.

\section{Neurophysiology and prevalence analysis}

Each patient had a 24-hour long ambulatory EEG without video monitoring as an inpatient in our hospital. We used 34 channels and $128 \mathrm{~Hz}$ sampling rate (Micromed Morpheus Polysomnograph, 10-20 electrode placement system). For visual EEG analysis we used a bipolar montage, $10 \mathrm{microV} / \mathrm{mm}$ amplification, $70 \mathrm{~Hz}$ high pass-, $0.53 \mathrm{~Hz}$ low pass-, and $50 \mathrm{~Hz}$ notch filters. We focused on the recognition of IEDs since their presence together with a previous epileptic seizure supports the diagnosis of epilepsy [34]. IEDs were defined as paroxysmal EEG graphoelements (spikes or sharp waves) of 20-200 ms duration followed by slow waves and disrupted background activity. Two independent, trained neurophysiologists analyzed the EEGs (Z. Cs., A. H.). One of them was blinded to the identity of the patients, while the other one knew the clinical history. A graphoelement was considered as IED (localized or generalized spikes or sharp waves; spike-and-wave complexes, $\geq 3$ s rhythmic synchronization) [35], if both raters evaluated it so. To analyze the sensitivity of ambulatory EEG in the diagnosis of AD-related epilepsy, we examined the occurrence of IEDs in the first 30 min of recordings (the length of a routine daytime EEG) separately.

The diagnosis of epilepsy was based on the current ILAE recommendations [18]; two epileptic seizures with at least 24 hours apart or one unprovoked seizure with interictal epileptiform activity on the EEG. Epileptologic data were collected retrospectively recalled from self or by others and from previous medical records. Seizure semiology was determined by three experienced epileptologists (A.SZ., G.B., A.K.). The classification of seizures was based on the current ILAE guidelines; the semiological features were described accordingly. A seizure with motor action of at least one limb was considered convulsive. A seizure was considered as generalized if the jerks involved all limbs. An aware focal seizure was defined as an episode with focal symptoms and preserved consciousness, while by definition, during a focal seizure with impaired awareness disturbance of consciousness or cognitive distraction of the patient was recognized.

\section{Risk analysis}

We divided the AD patients into three subgroups; one with epileptic seizures (AD+ES), one with epileptiform discharges $(\mathrm{AD}+\mathrm{ED})$ and another with no epilepsy $(\mathrm{AD}+\mathrm{NE})$. Intergroup differences were compared using Kruskal-Wallis or independent sample ANOVA analysis. Since epileptiform potentials might indicate the presence of epilepsy but the association is not clear in $\mathrm{AD}$, we compared the clinical and epidemiological data of the AD+ES and AD+ED using $t$-tests and Mann-Whitney $\mathrm{U}$ test. We applied IBM SPSS 20 software for statistics.

According to the literature, the level of education, the severity of temporal and frontal atrophy, the 


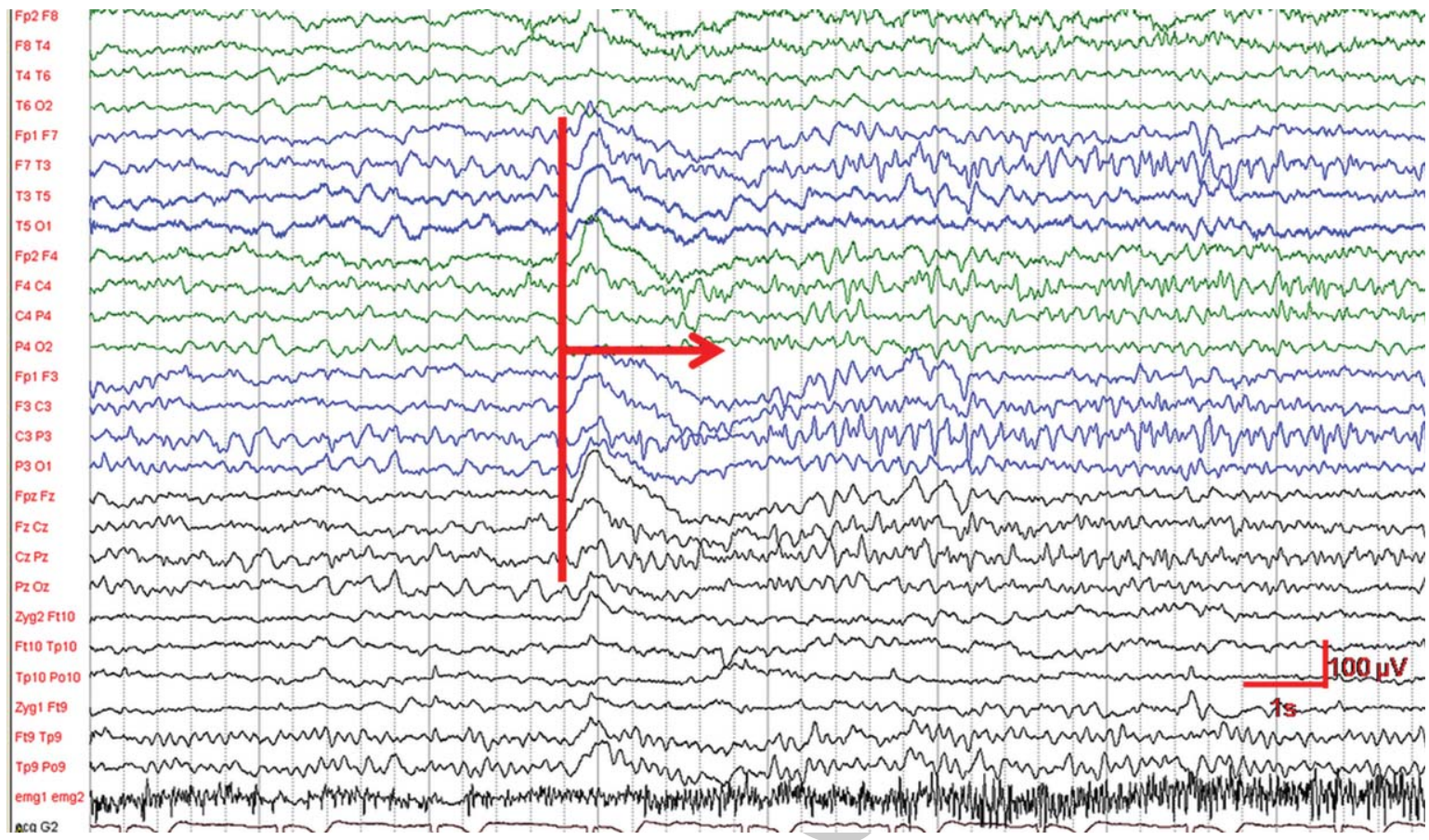

Fig. 1. EEG section from a 24-hour EEG recording of an AD patient. The figure demonstrates the build-up of an epileptic ictal activity in the left temporal and centro-parietal electrodes (arrow).

onset of cognitive decline and the severity of the disease represent risk factors for dementia-associated epilepsy; these features were analyzed as potential predictors using multivariate logistic regression analysis. We measured the education level with years of schooling, the atrophy level with the MTA score and the disease severity with the ACE score. Since many studies demonstrated that AD patients have a high risk for epileptic seizures but reports on other type of dementias are limited, we hypothetically proposed that the pattern of cognitive decline measured by ACE indicating Alzheimer-type dementia might predict the presence of seizures, so we added the VLOM ratio to the model as well. Confidence intervals (95\%), prevalence estimates and point estimates (Odds-ratio) were calculated by logistic regression.

\section{RESULTS}

\section{Demographic and prevalence data}

In twenty patients out of the forty-two (48\%) we did not detect epileptiform activity or seizures on the EEG, neither found any data on seizures in the past medical history. In twelve patients $(28 \%)$ we found epileptiform discharges on the EEG but there were no seizures in the history. We diagnosed epileptic seizures in ten patients out of forty-two (24\%). In two patients of these ten, we recorded electric ictal pattern two times during the recording period (Fig. 1). In four patients of ten, two or more seizures were identified in the medical history, while in six patients only one (95\%CI: $0.99-2.61)$. In 8 of the $10 \mathrm{AD}$ patients with clinically identified seizures, the ambulatory EEG showed IEDs as well (95\%CI: 0.5-1.1) (Fig. 2). Altogether, we detected epileptiform activity in $48 \%$ of our $\mathrm{AD}$ patients ( 8 patients from $\mathrm{AD}+\mathrm{ES}$ group and 12 patients of $\mathrm{AD}+\mathrm{ED}$ group).

In the first $30 \mathrm{~min}$ the EEG contained IEDs in only three patients out of those twenty who had either seizures or IEDs in the full length recording ( 8 with seizures+ interictal pattern and 12 with epileptiform discharges); therefore, if we suppose that the first $30 \mathrm{~min}$ of the ambulatory recording corresponds to a routine EEG, than the calculated sensitivity value for routine EEG in this study is only $15 \%(95 \% \mathrm{CI}$ : $-0.02-0.32$ ).

We collected the major demographic and clinical features of the three analyzed groups: 1) $A D$ patients without seizures or subclinical epileptiform discharges (AD+NE; $n=20), 2)$ AD patients with epileptic seizures (AD+ES; $n=10)$, and 3$) \mathrm{AD}$ 


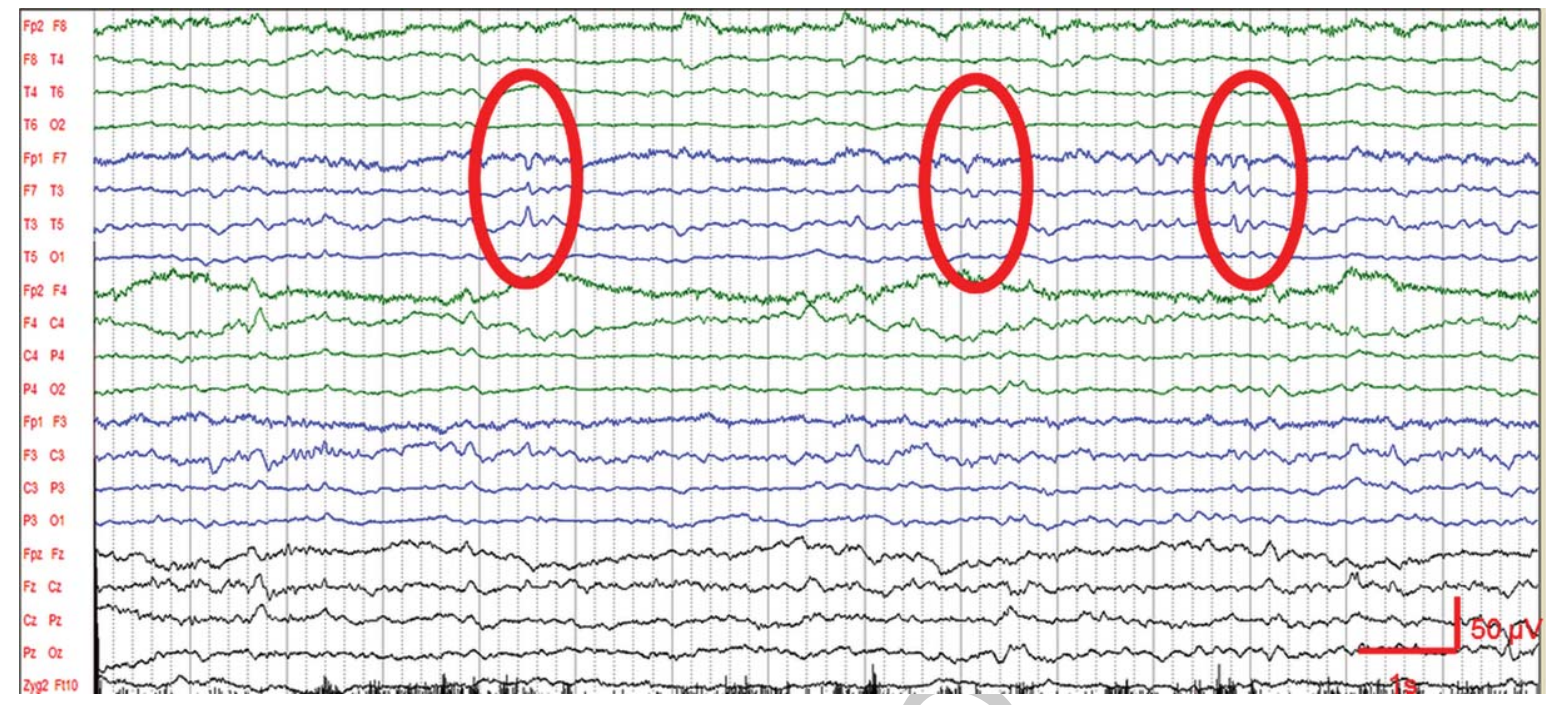

Fig. 2. EEG section from a 24-hour EEG recording of the same AD patient than in Fig. 1. There is an interictal sharp wave in the left temporal region with phase inversion at F7 electrode (circles).

patients without seizures but with epileptiform discharges (AD+ED; $n=12)$. Data were compared with Kruskal-Wallis and independent ANOVA analysis. The three groups did not differ significantly in gender and age; however, they showed differences in several clinical features as it is demonstrated in Table 1.

To analyze the value of EEG positivity in AD, we compared the $\mathrm{AD}+\mathrm{ED}$ and the $\mathrm{AD}+\mathrm{ES}$ groups with univariate analysis. The differences between the $\mathrm{AD}+\mathrm{ES}$ and $\mathrm{AD}+\mathrm{ED}$ groups were non-significant for age (95\%CI: $-4.43-9.065$; p: 0.482$)$, for the age of onset (95\% CI: $-7.78-7.44$; p: 0.964), for education level (95\% CI: $-0.671-1.83$; p: 0.343), for MTA score (95\%CI: $-0.273-0.673$; p: 0.388), for sex (p: 0.353), for handedness (p: 0.658), or for memantine therapy (p: 0.844). The only marginal difference was the duration of dementia (95\%CI: 0.16-4.8, p: 0.037), where patients with seizures showed longer duration.

\section{Risk factors for epileptic activity revealed by} logistic regression analysis

Since the AD+ED and the AD+ES groups showed many similarities, in order to increase the statistical power, we combined the $\mathrm{AD}+\mathrm{ES}$ and $\mathrm{AD}+\mathrm{ED}$ groups (AD+ES/ED, altogether 22 patients) and compared the data to the $\mathrm{AD}+\mathrm{NE}$ group (20 patients). In a logistic regression model, we analyzed which potential

Table 1

Demographic and clinical features of AD patients with epileptic seizures (AD+ES), with epileptiform discharges (AD+ED) and without epileptic seizures or EEG positivity (AD+NE) and the results of statistical comparisons

\begin{tabular}{lcccc}
\hline Parameter & AD+NE & AD+ES & AD+ED & $\begin{array}{c}\text { Results of ANOVA } \\
\text { and Kruskal-Wallis }\end{array}$ \\
\hline Number of patients & & & 12 & - \\
Gender (\% of females) & 20 & $70 \%$ & $60 \%$ & 0.94 \\
Memantine therapy (\% of patients) & $15 \%$ & $20 \%$ & $17 \%$ & 0.117 \\
Cholinesterase inhibitor therapy (\% of patients) & $100 \%$ & $100 \%$ & $100 \%$ & 1 \\
Age & $79.4 \pm 7.4$ & $75.9 \pm 4.7$ & $73.6 \pm 9.3$ & 0.110 \\
Handedness (\% of right handed patients) & $86 \%$ & $90 \%$ & $84 \%$ & 0.209 \\
MTA score & $2.65 \pm 0.67$ & $3.2 \pm 0.78$ & $3 \pm 0.6$ & $* 0.02$ \\
Age at the onset of dementia & $74.4 \pm 5.9$ & $68.5 \pm 4.1$ & $68.6 \pm 10.9$ & $* 0.049$ \\
Duration of dementia & $4.9 \pm 2.9$ & $7.4 \pm 1.7$ & $4.91 \pm 3.14$ & $* 0.049$ \\
Education (y) & $11.6 \pm 2.5$ & $16.5 \pm 1.1$ & $15.9 \pm 1.6$ & $*<0.001$ \\
ACE score & $67.4 \pm 9.4$ & $38.4 \pm 15.3$ & $44.5 \pm 7.4$ & $*<0.001$ \\
VLOM ratio & $3.3 \pm 0.8$ & $3.5 \pm 0.2$ & $3.5 \pm 0.1$ & $* 0.008$ \\
\hline
\end{tabular}

* indicates statistical significance between the three analyzed groups. MRI supported the diagnosis of AD in every case based on the MTA score. MTA, medial temporal lobe atrophy score; ACE, Addenbrooke Cognitive Examination; VLOM, verbal-language/orientation-memory ratio. 
Table 2

Results of logistic regression analysis demonstrating Odds-ratios and confidence intervals

\begin{tabular}{lcc}
\hline Variable & Odds-ratio & 95\% confidence interval \\
\hline Age (y) & 0.973 & $0.514-1.839$ \\
Onset of dementia (age) & 0.991 & $0.48-2.047$ \\
Education (y) & 1.801 & $0.875-3.706$ \\
MTA score & 1.095 & $0.026-45.56$ \\
ACE score & 0.874 & $0.761-1.003$ \\
VLOM ratio & 26.77 & $0.036-20169$ \\
\hline
\end{tabular}

* indicates significant differences. ACE, Addenbrooke Cognitive Examination; MTA, medial temporal lobe atrophy score; VLOM, verbal-language/memory-orientation ratio.

factors increase the risk of developing epileptic activity. We found that patients with higher education (odds ratio for 1 year higher education: 1.8), higher ACE scores (odds ratio for 1 point decrease: 0.87 ) and increased VLOM ratios (odds ratio: 2.68 for 0.1 point) have an increased chance for developing epileptic activity (Table 2).

\section{Seizure semiology}

We analyzed the semiologic features of eighteen seizures of ten $\mathrm{AD}$ patients based on the auto-, heteroanamnesis and previous medical reports (Table 3 ). We detected generalized tonic-clonic seizures in $11 \%$ and focal onset seizures with impaired awareness in $72 \%$. Fifty-five percent of seizures were non-motor.

\section{DISCUSSION}

Growing body of evidence suggests that AD patients have a high risk to develop epileptic seizures [5]. However, the real prevalence of epilepsy is not known due to the methodological differences of human prevalence studies and to the limited number of studies analyzing the risk factors of AD-related epileptic seizures.
Most of the previous studies used retrospective analyses from hospital based or national databanks [36-40]. The major limitation of these studies is the difficulty to prove the diagnosis of epilepsy or AD in questionable cases. The comparison of our results with previous data is also hard, due to the changes in the diagnostic guidelines and criteria of $\mathrm{AD}$ [17]. However, most prevalence studies found elevated risk for epileptic seizures [5] being in line with our current results where $24 \%$ of $\mathrm{AD}$ patients have epileptic seizures and another $28 \%$ shows epileptic activity on the EEG.

EEG is essential in the diagnosis of epilepsy according to the existing guidelines [18]. Longterm EEG recordings including sleep may offer an importantly higher diagnostic yield [41], as we have reported earlier [42]. Our current research demonstrates that 24-hour ambulatory EEG is a safe and well tolerated method for the diagnosis of AD-related epileptic activity since all recordings were performed without any complications. In the literature only one study applied EEG using mostly only routine $30 \mathrm{~min}$ recordings and a few serial EEGs [24]. The current study also reinforces that short EEGs have a low diagnostic yield, as we found only $15 \%$ sensitivity for IEDs of the first $30 \mathrm{~min}$ EEG which is the average length of a routine EEG recording. It demonstrates an evidence for the limitation of brief EEG monitoring in the clinical assessment of dementia associated epilepsy. The benefit of extension the length of EEG over 24 hours is questionable in $\mathrm{AD}$; however, our previous results indicated that the sensitivity of EEG reaches a plateau after 8-hour sleep EEG [42].

The remarkable similarity of demographic and clinical data of the patients with epileptic seizures $(\mathrm{AD}+\mathrm{ES})$ and the patients with epileptiform discharges only (AD+ED) is a very important finding. There are only limited number of studies investigating the prevalence and importance of epileptiform

Table 3

The semiology of epileptic seizures in AD patients. Seizures were focal with impaired awareness in $72 \%$ and in $55 \%$ there was no motor activity seen

\begin{tabular}{lcc}
\hline Type of seizure & $\begin{array}{c}\text { Number of } \\
\text { seizures }\end{array}$ & Semiology features \\
\hline $\begin{array}{l}\text { Aware focal } \\
\text { Non-motor }\end{array}$ & 1 & $\begin{array}{c}\text { Jamais vu/déja vu } \\
\text { Motor }\end{array}$ \\
$\begin{array}{l}\text { Impaired awareness focal } \\
\text { Non-motor }\end{array}$ & 1 & Clonization of right upper limb \\
$\begin{array}{l}\text { Motor } \\
\text { Generalized }\end{array}$ & 8 & Speech arrest (1), amnestic episode (4), confusion (3) \\
Atonic & 5 & Bilateral limb jerks (3), Right upper limb clonus (2) \\
Tonic-clonic & 1 & Atonia \\
\hline
\end{tabular}


potentials in $\mathrm{AD}$ with ambiguous prevalence data. Liedorp and his colleagues analyzed the data of 30 min long daytime EEGs of 1674 AD patients and found subclinical epileptiform activity only in $3 \%$ of the sample [43]. In Vossel's study involving serial routine EEG recordings, the prevalence of epileptiform activity was 6\% [24]. Our finding of $48 \%$ epileptiform activity rate is significantly higher. The explanation might be that we analyzed 24 hours long EEGs including whole night sleep recordings. It has been clearly shown in several epilepsy studies that epileptiform activity increases during sleep. We have reported similar findings in AD patients [42]. In the study of Vossel et al., the routine EEG was normal in $60 \%$ of those AD patients who had clinically overt epileptic seizures, while sleep deprived serial EEGs have shown epileptiform discharges in $84 \%$ in those patients [24]. This is supported by another study of Vossel's group about the temporal distribution of epileptiform activity in AD showing that epileptic activity occurs almost exclusively (90\%) during sleep [26]. They found epileptiform discharges in $42 \%$ of $\mathrm{AD}$ patients being in line with our results. Additionally, those patients suffered 2.5-times faster decline in their yearly measured MMSE scores than patients with no epileptic activity suggesting the significant importance of epileptiform activity in the progression of $\mathrm{AD}$.

Our results show strong link between the patients with definitive seizures (AD+ES) and with epileptiform activity (AD+ED). Based on the overlapping clinical, demographical features and on the knowledge from previous studies suggesting that epileptiform discharges are associated with accelerated cognitive decline, we propose that the AD+ED patient group represents incidental new epilepsy cases. While further studies are required to reinforce these findings, we suggest that we might consider thinking about AD patients with epileptiform EEG as patients with epilepsy.

A further point is analysis of seizure semiology which has been performed in only four reports in the literature. One of them was a prevalence study [24], while the others examined dementia patients with known epilepsy diagnosis [38, 44, 45]. These three studies analyzed inhomogeneous patient groups, where out of the altogether 167 patients only 108 had AD. The four studies reported generalized tonicclonic seizures in $32.5 \%$, focal seizures with impaired consciousness in $52.25 \%$ and aware focal seizures in $15 \%$. Based on the literature, non-motor seizures with impaired awareness are typical among AD patients.
In the most detailed study, the common symptoms were confusion, disturbance of consciousness, jamais vu, déjà vu, and visceral sensations [24]; all typical in temporal lobe epilepsy [46]. In our study, generalized tonic-clonic seizures were less frequent $(11 \%)$, while we found focal seizures with impaired consciousness in $(72 \%)$ mostly without motor activity; being in line with previous findings [24, 38, 44, 45].

The dominance of focal non-motor seizures with impaired awareness raises an important clinical consideration. AD patients frequently show daily changes in mood, in cognitive performance and in communication skills. In light of the present study, this variability might at least partially be explained by epileptic disturbances. Video-EEG monitoring or ultra-long-term EEG recordings are needed to clarify the epileptic background of these phenomena. We can conclude that it is challenging to recognize the symptoms of non-motor focal seizures in $\mathrm{AD}$ and without searching for them, epilepsy may easily remain hidden $[47,48]$.

Early studies regarding the risk factors of ADrelated epileptic seizures were not conclusive [14, 23]. Amatniek et al. found that more severe disease stage (relative risk: 4.15), early onset of cognitive decline (relative risk: 0.89/year), African American race (relative risk: 7.35) and epileptiform EEG signals (relative risk: 73.36) were important risk factors [22]. Early disease onset as a risk factor has also been shown [36, 49]. Volicer et al. demonstrated the impact of severe stage of $\mathrm{AD}$ [50], while in other studies the incidence of seizures was independent from the disease stage [45, 51]. Vossel and his coworkers reported that seizures were present also in less advanced disease, even in mild cognitive impairment [24]. The same study demonstrated that higher education is also a risk factor. To our best knowledge, the neuropsychological pattern of cognitive dysfunction represented in our study by the VLOM ratio has been not analyzed in previous reports.

The early onset of cognitive decline is a consistent risk factor of epilepsy in the literature. A possible explanation is that early onset might be related to a genetic cause, and it is known that in these forms, epileptic seizures are significantly more frequent [13]. In our study this factor was not statistically significant, however, in line with some previous reports, the severe disease state was a prominent risk factor (odds ratio: 0.874 for 1 point ACE score increase) $[22,50]$. Regression analysis has not been applied in previous publications, so it might be possible that the leading risk factor is the severe disease stage and 
patients with earlier onset reach the advanced disease state sooner. However, seizures were reported in the preclinical and mild stages as well [24, 44, 47]. In the future, we aim to answer this question by studies involving patients with mild AD and mild cognitive impairment.

To our best knowledge, there is no previous study analyzing the neuropsychological pattern as a seizure predicting factor in dementia. We found that the elevated VLOM ratio has a 26.77 odds ratio for epilepsy in AD. It might be possible that the increased VLOM ratio is connected to the more severe disease stage, so the typical neuropsychological pattern became more prominent during the development of cognitive decline. However, it is also possible that AD is a predisposing disease for epilepsy compared to other neurocognitive disorders. Therefore, the Alzheimer-like VLOM ratio could be a good indicator for physicians to search for concomitant epilepsy, especially if we consider that 0.1 point increase in the VLOM ratio is associated to 2.67 odds ratio for developing seizures. Further studies analyzing the occurrence of epilepsy in AD patients with typical and atypical VLOM pattern from the early phase of cognitive deterioration are needed to reinforce these findings.

Epileptiform EEG is a well-known indicator of seizure activity in epileptology. In our study AD patients with epileptiform potentials showed seizures in $40 \%$ (eight $\mathrm{AD}$ patients with seizures and EDs out of twenty AD patients (eight AD patients with seizures and EDs+twelve with EDs only), while just $8 \%$ had no epileptiform discharges (two AD patients with seizures and without IEDs out of the twenty-two AD patients (two AD patients with seizures and without IEDs+twenty patients without IEDs or seizures). Thus, we confirmed the findings of Amatniek et al. in AD [22]: epileptiform EEG indicates well the presence of seizures in AD.

The higher education as a risk factor (odds ratio: 1.8 ) is curious, however, it is consistent with the results of Vossel et al. [24]. It seems feasible that patients with higher education report their symptoms more accurately. However, it has also been consistently illustrated that $\mathrm{AD}$ patients with higher education present faster cognitive decline [52, 53]. Cognitive reserve hypothesis could be a possible explanation, so higher educated patients are able to compensate the cognitive loss for a longer period, so at the moment of the diagnosis, the disease will terminate in a shorter time [53].

Amyloid is an evolutionary preserved molecule having a crucial role in memory and learning functions [54]. Theoretically, it is possible that higher cognitive functioning might augment the amyloid burden. Amyloid has an epileptogenic potential [55] and vice versa, epileptic activity increases the amyloid deposition via the hyperexcitation [25]. Because the clearance mechanisms are impaired in $\mathrm{AD}$, amyloid accumulation might lead to frequent epileptic activity, which in turn, might accelerate the progression of the disease in highly educated AD patients. Animal models [25] and human studies support this mechanism [26]. Thus, concomitant epilepsy might be an explanation both for the educational risk of seizures and for the education as a modifying factor in the progression. However, in our sample patients with epilepsy showed earlier cognitive decline and higher education seeming to be contradictory, especially if we consider that the average age for the onset of dementia $(\sim 69)$ is a relatively early age in most of the dementia populations. The inconsistency might be explainable with the clinical sample oversampling the care-recipients. Population-based studies are clearly more representative; however, to define a homogenous AD sample and to exclude the effect of applied medication on the epileptic threshold, we had to use all of the available clinical data. Thus, we have to avoid generalizations and have to emphasize that the higher education is still a questionable risk factor for AD-related epilepsy. Further prospective longitudinal neurophysiologic studies are needed to answer this question.

\section{Conclusions}

We found epileptic seizures in a quarter of $\mathrm{AD}$ patients and epileptiform activity in $48 \%$ of them; therefore, we can conclude that epilepsy represents an important comorbidity of AD. Patients with seizures and patients with epileptiform discharges show similar clinical characteristics suggesting that EEG positivity in AD might represent new incidentally diagnosed epilepsy cases. The typical seizures are non-motor focal seizures which are hard to recognize without the application of a sensitive EEG technique. This might be one of the reasons why seizures and epilepsy are underdiagnosed in AD.

The 24-hour ambulatory EEG is a safe tool to increase diagnostic accuracy of epilepsy in AD. Patients with higher education, with AD-type neuropsychological pattern (typical VLOM ratio) and in a more severe disease stage have a higher chance to develop epileptic seizures and epileptiform activity. Thus, ACE score and VLOM ratio represent a 
useful clinical tool to identify patients with high risk for epilepsy. Once epilepsy is diagnosed in this patient group antiepileptic drugs might be considered as a promising new therapeutic approach. However, further studies are needed to measure the usefulness of antiepileptic drugs in AD and additional prevalence studies are necessary to examine the occurrence of seizures in the early or prodromal phases of AD.

\section{ACKNOWLEDGMENTS}

Our research was supported by the National Brain Research Program (KTIA_NAP_13-1-2013-0001; 2017-1.2.1-NKP-2017-00002) and MET Hungary Ltd. Special thanks to Zita Csepella for analyzing the EEG recordings.

Authors' disclosures available online (https:// www.j-alz.com/manuscript-disclosures/17-0925r3).

\section{REFERENCES}

[1] Prince M, Bryce R, Albanese E, Wimo A, Ribeiro W, Ferri CP (2013) The global prevalence of dementia: A systematic review and metaanalysis. Alzheimers Dement 9, 63-75.

[2] Ferri CP, Prince M, Brayne C, Brodaty H, Fratiglioni L, Ganguli M, Hendrie H, Huang Y, Jorm A, Mathers C, Menezes PR, Rimmer E, Scazufca M, Alzheimer's Disease International (2006) Global prevalence of dementia: A Delphi consensus study. Lancet 366, 2112-2117.

[3] Harvey R, Skelton-Robinson M, Rossor M (2003) The prevalence and causes of dementia in people under the age of 65 years. J Neurol Neurosurg Psychiatry 74, 1206-1209.

[4] Kivipelto M, Solomon A, Ahtiluoto S, Ngandu T, Lehtisalo J, Antikainen R, Bäckman L, Hänninen T, Jula A, Laatikainen T, Lindström J, Mangialasche F, Nissinen A, Paajanen T, Pajala S, Peltonen M, Rauramaa R, StigsdotterNeely A, Strandberg T, Tuomilehto J, Soininen H (2013) The Finnish geriatric intervention study to prevent cognitive impairment and disability (FINGER): Study design and progress. Alzheimers Dement 9, 657-665.

[5] Horváth A, Szucs A, Barcs G, Noebels JL, Kamondi A (2016) Epileptic seizures in Alzheimer disease: A review. Alzheimer Dis Assoc Disord 30, 186-192.

[6] Puvenna V, Engeler M, Banjara M, Brennan C, Schreiber P, Dadas A, Bahrami A, Solanki J, Bandyopadhyay A, Morris JK, Bernick C, Ghosh C, Rapp E, Bazarian JJ, Janigro D (2016) Is phosphorylated tau unique to chronic traumatic encephalopathy? Phosphorylated tau in epileptic brain and chronic traumatic encephalopathy. Brain Res $\mathbf{1 6 3 0}$, 225-240

[7] Tai XY, Koepp M, Duncan JS, Fox N, Thompson P, Baxendale S, Liu JY, Reeves C, Michalak Z, Thom M (2016) Hyperphosphorylated tau in patients with refractory epilepsy correlates with cognitive decline: A study of temporal lobe resections. Brain 139, 2441-2455.

[8] Kato T, Inui Y, Nakamura A, Ito K (2016) Brain fluorodeoxyglucose (FDG) PET in dementia. Ageing Res Rev 30, 73-84.
[9] Walker MC (2015) Hippocampal sclerosis: Causes and prevention. Semin Neurol 35, 193-200.

[10] Riascos D, Nicholas A, Samaeekia R, Yukhananov R, Mesulam MM, Bigio EH, Weintraub S, Guo L, Geula C (2014)Alterations of $\mathrm{Ca}(2)(+)$-responsive proteins within cholinergic neurons in aging and Alzheimer's disease. Neurobiol Aging 35, 1325-1333.

[11] Palop JJ, Chin J, Roberson ED, Wang J, Thwin MT, Bien-Ly N, Yoo J, Ho KO, Yu GQ, Kreitzer A, Finkbeiner S, Noebels JL, Mucke L (2007) Aberrant excitatory neuronal activity and compensatory remodeling of inhibitory hippocampal circuits in mouse models of Alzheimer's disease. Neuron 55, 697-711.

[12] Westmark CJ, Westmark PR, Beard AM, Hildebrandt SM, Malter JS (2008) Seizure susceptibility and mortality in mice that over-express amyloid precursor protein. Int J Clin Exp Pathol 1, 157.

[13] Mendez MF, Catanzaro P, Doss RC, Arguello R, Frey WH (1994) Seizures in Alzheimer's disease: Clinicopathologic study. J Geriatr Psychiatry Neurol 7, 230-233.

[14] Hauser WA, Morris ML, Heston LL, Anderson VE (1986) Seizures and myoclonus in patients with Alzheimer's disease. Neurology 36, 1226-1226.

[15] Risse SC, LampeTH, Bird TD, Nochlin D, Sumi SM, Keenan T, Cubberley L, Peskind E, Raskind MA (1990) Myoclonus, seizures, and paratonia in Alzheimer disease. Alzheimer Dis Assoc Disord 4, 217-225.

[16] Cabrejo L, Guyant-Maréchal L, Laquerrière A, Vercelletto M, De La Fournière F, Thomas-Antérion C, Verny C, Letournel F, Pasquier F, Vital A, Checler F (2006) Phenotype associated with APP duplication in five families. Brain 129, 2966-2976.

[17] McKhann GM, Knopman DS, Chertkow H, Hyman BT, Jack CR, Kawas CH, Klunk WE, Koroshetz WJ, Manly JJ, Mayeux R, Mohs RC, Morris JC, Rossor MN, Scheltens P, Carrillo MC, Thies B, Weintraub S, Phelps $\mathrm{CH}$ (2011)The diagnosis of dementia due to Alzheimer's disease: Recommendations from the National Institute on Aging-Alzheimer's Association workgroups on diagnostic guidelines for Alzheimer's disease. Alzheimers Dement 7, 263-269.

[18] Fisher RS, Acevedo C, Arzimanoglou A, Bogacz A, Cross JH, Elger CE, Engel J Jr, Forsgren L, French JA, Glynn M, Hesdorffer DC, Lee BI, Mathern GW, Moshé SL, Perucca E, Scheffer IE, Tomson T, Watanabe M, Wiebe S (2014) ILAE official report: A practical clinical definition of epilepsy. Epilepsia 55, 475-482.

[19] Blinowska KJ, Rakowski F, Kaminski M, Fallani FDV, Del Percio C, Lizio R, Babiloni C (2017) Functional and effective brain connectivity for discrimination between Alzheimer's patients and healthy individuals: A study on resting state EEG rhythms. Clin Neurophysiol 128, 667-680.

[20] Babiloni C, Lizio R, Marzano N, Capotosto P, Soricelli A, Triggiani AI, Del Percio C (2016) Brain neural synchronization and functional coupling in Alzheimer's disease as revealed by resting state EEG rhythms. Intern J Psychophysiol 103, 88-102.

[21] Horvath A, Szucs A, Csukly G, Sakovics A, Stefanics G, Kamondi A (2018) EEG and ERP biomarkers of Alzheimer's disease: A critical review. Front Biosci 23, 183-220.

[22] Amatniek JC, Hauser WA, DelCastillo-Castaneda C, Jacobs DM, Marder K, Bell K, Albert M, Brandt J, Stern Y (2006) Incidence and predictors of seizures in patients with Alzheimer's disease. Epilepsia 47, 867-872. 
[23] Romanelli MF, Morris JC, Ashkin K, Coben LA (1990) Advanced Alzheimer's disease is a risk factor for late-onset seizures. Arch Neurol 47, 847-850.

[24] Vossel KA, Beagle AJ, Rabinovici GD, Shu H, Lee SE, Naasan G, Hegde M, Cornes SB, Henry ML, Nelson AB, Seeley WW, Geschwind MD, Gorno-Tempini ML, Shih T, Kirsch HE, Garcia PA, Miller BL, Mucke L (2013) Seizures and epileptiform activity in the early stages of Alzheimer disease. JAMA Neurol 70, 1158-1166.

[25] Dolev I, Fogel H, Milshtein H, Berdichevsky Y, Lipstein N, Brose N, Gazit N, Slutsky I (2013) Spike bursts increase amyloid-beta $40 / 42$ ratio by inducing a presenilin-1 conformational change. Nat Neurosci 16, 587-595.

[26] Vossel KA, Ranasinghe KG, Beagle AJ, Mizuiri D, Honma SM, Dowling AF, Darwish SM, Van Berlo V, Barnes DE, Mantle M, Karydas AM, Coppola G, Roberson ED, Miller BL, Garcia PA, Kirsch HE, Mucke L, Nagarajan SS (2016) Incidence and impact of subclinical epileptiform activity in Alzheimer's disease. Ann Neurol 80, 858-870.

[27] Noebels J (2011) A perfect storm: Converging paths of epilepsy and Alzheimer's dementia intersect in the hippocampal formation. Epilepsia 52, 39-46.

[28] Duara R, Loewenstein DA, PotterE, Appel J, Greig MT, Urs R, Schofield E (2008) Medial temporal lobe atrophy on MRI scans and the diagnosis of Alzheimer disease. Neurology 71, 1986-1992.

[29] Laakso MP, Frisoni GB, Könönen M, Mikkonen M, Beltramello A, Geroldi C, Bianchetti A, Trabucchi M, Soininen $\mathrm{H}$, Aronen HJ (2000) Hippocampus and entorhinal cortex in frontotemporal dementia and Alzheimer's disease: A morphometric MRI study. Biol Psychiatry 47, 1056-1063.

[30] Vossel KA, Tartaglia MC, Nygaard HB, Zeman AZ, Miller BL (2017) Epileptic activity in Alzheimer's disease: Causes and clinical relevance. Lancet Neurol 16, 311-322.

[31] Dudas RB, Berrios GE, Hodges JR (2000) The Addenbrooke's cognitive examination (ACE) in the differential diagnosis of early dementias versus affective disorder. Am $J$ Geriatr Psychiatry 13, 218-226.

[32] Cockrell JR, Folstein MF (2002) Mini-Mental State Examination. In Principles and Practice of Geriatric Psychiatry, Second Edition, Copeland JRM, Abou-Saleh MT, Blazer DG, eds. John Wiley \& Sons, Ltd, Chichester, UK.

[33] Torralva T, Roca M, Gleichgerrcht E, Bekinschtein T, Manes F (2009) A neuropsychological battery to detect specific executive and social cognitive impairments in early frontotemporal dementia. Brain 132, 1299-1309.

[34] Engel J Jr (2001) Mesial temporal lobe epilepsy: What have we learned? Neuroscience 7, 340-352.

[35] Noachtar S, Rémi J (2009) The role of EEG in epilepsy: A critical review. Epilepsy Behav 15, 22-33.

[36] Irizarry MC, Jin S, He F, Emond JA, Raman R, Thomas RG, Sano M, Quinn JF, Tariot PN, Galasko DR, Ishihara LS, Weil JG, Aisen PS (2012) Incidence of new-onset seizures in mild to moderate Alzheimer disease. Arch Neurol 69, 368-372.

[37] Sherzai D, Losey T, Vega S, Sherzai A (2014) Seizures and dementia in the elderly: Nationwide Inpatient Sample 1999-2008. Epilepsy Behav 36, 53-56.

[38] Rao SC, Dove G, Cascino GD, Petersen RC (2009) Recurrent seizures in patients with dementia: Frequency, seizure types, and treatment outcome. Epilepsy Behav 14, 118-120.

[39] Imfeld P, Bodmer M, Schuerch M, Jick SS, Meier CR (2013) Seizures in patients with Alzheimer's disease or vascular dementia: A population-based nested case-control analysis. Epilepsia 54, 700-707.

[40] Lozsadi D, Larner A (2006) Prevalence and causes of seizures at the time of diagnosis of probable Alzheimer's disease. Dement Geriatr Cogn Disord 22, 121-124.

[41] Smith S (2005) EEG in the diagnosis, classification, and management of patients with epilepsy. J Neurol Neurosurg Psychiatry 76, ii2-ii7.

[42] Horvath A, Szucs A, Barcs G, Kamondi A (2017) Sleep EEG detects epileptiform activity in Alzheimer's disease with high sensitivity. J Alzheimers Dis 56, 1175-1183.

[43] Liedorp M, Stam CJ, Van Der Flier WM, Pijnenburg YA, Scheltens P (2010) Prevalence and clinical significance of epileptiform EEG discharges in a large memory clinic cohort. Dement Geriatr Cogn Disord 29, 432-437.

[44] Cretin B, Sellal F, Philippi N, Bousiges O, Di Bitonto L, Martin-Hunyadi C, Blanc F (2016) Epileptic prodromal Alzheimer's disease, a retrospective study of 13 new cases: Expanding the spectrum of Alzheimer's disease to an epileptic variant? J Alzheimers Dis 52, 1125-1133.

[45] Sarkis RA, Dickerson BC, Cole AJ, Chemali ZN (2016) Clinical and neurophysiologic characteristics of unprovoked seizures in patients diagnosed with dementia. J Neuropsy Clin Neurosci 28, 56-61.

[46] Wieser H-G (2004) ILAE Commission Report. Mesial temporal lobe epilepsy with hippocampal sclerosis. Epilepsia 45, 695-714.

[47] Lam AD, Deck G, Goldman A, Eskandar EN, Noebels J, Cole AJ (2017) Silent hippocampal seizures and spikes identified by foramen ovale electrodes in Alzheimer's disease. Nat Med 23, 678-680.

[48] Horvath A, Szucs A, Barcs G, Fabo D, Kelemen A, Halasz P, Eross L, Kamondi A (2017) Interictal epileptiform activity in the foramen ovale electrodes of a non-epileptic frontotemporal dementia patient. J Alzheimer Dis Rep 1, 89-96.

[49] Scarmeas N, Honig LS, Choi H, Cantero J, Brandt J, Blacker D, Albert M, Amatniek JC, Marder K, Bell K, Hauser WA, Stern Y (2009) Seizures in Alzheimer disease: Who, when, and how common? Arch Neurol 66, 992-997.

[50] Volicer L, Smith S, Volicer BJ (1995) Effect of seizures on progression of dementia of the Alzheimer type. Dement Geriatr Cogn Disord 6, 258-263.

[51] Hesdorffer D, Hauser W, Annegers J, Kokmen E, Rocca WA (1996) Dementia and adult-onset unprovoked seizures. Neurology 46, 727-730.

[52] Musicco M, Palmer K, Salamone G, Lupo F, Perri R, Mosti S, Spalletta G, di Iulio F, Pettenati C, Cravello L, Caltagirone C (2009) Predictors of progression of cognitive decline in Alzheimer's disease: The role of vascular and sociodemographic factors. J Neurol 256, 1288.

[53] Ngandu T, von Strauss E, Helkala EL, Winblad B, Nissinen A, Tuomilehto J, Soininen H, Kivipelto M (2007) Education and dementia: What lies behind the association? Neurology 69, 1442-1450.

[54] Garcia-Osta A, Alberini CM (2009) Amyloid beta mediates memory formation. Learn Mem 16, 267-272.

[55] Busche MA, Eichhoff G, Adelsberger H, Abramowski D, Wiederhold K-H, Haass C, Staufenbiel M, Konnerth A, Garaschuk O (2008) Clusters of hyperactive neurons near amyloid plaques in a mouse model of Alzheimer's disease. Science 321, 1686-1689. 